declared, Isabel de la Morena Speakers bureau: Abbvie, Celgene, Pfzier, UCB, Ghebro, Roche, Sanofi, Janssen., J Fiter-Areste: None declared, Vega Jovani: None declared, A Martínez-Cristóbal : None declared, Lourdes Mateo: None declared, Sergi Ordoñez : None declared, D ReinaSanz: None declared, C Vergara-Dangond: None declared, V NúñezMonje: None declared, I Torner-Hernández: None declared, Juanjo J Alegre-Sancho: None declared

DOI: 10.1136/annrheumdis-2019-eular.5992

\section{FRI0683 DEFINING THE GUT MICROBIOME IN PATIENTS WITH ANCA-ASSOCIATED VASCULITIS}

catherine najem ${ }^{1,2}$, Jung-Jin Lee ${ }^{2}$, Ceylan Tanes ${ }^{2}$, Antoine Sreih ${ }^{2}$, Rennie Rhee ${ }^{2}$, Abdallah Geara ${ }^{2}$, LI Hongzhe ${ }^{2}$, Kyle Bittinger ${ }^{2}$, James Lewis ${ }^{2}$, Peter Merkel ${ }^{2}$.

${ }^{1}$ Temple University Hospital, Rheumatology, Philadelphia, United States of

America; ${ }^{2}$ University of Pennsylvania, Philadelphia, United States of America

Background: Although a link between gut microbiome and autoimmune diseases has been suggested, there is a gap in the understanding of the gut microbiome in ANCA-associated vasculitis (AAV).

Objectives: This study evaluated the gut microbiome in AAV (granulomatosis with polyangiitis (GPA), microscopic polyangiitis, and eosinophilic granulomatosis with polyangiitis) compared to healthy controls.

Methods: Using cross-sectional and longitudinal designs, the gut microbiome was compared among patients with i) newly-diagnosed AAV (active and remission); ii) chronic AAV (active and remission), and iii) healthy controls. Fecal samples were collected using standardized methods and analyzed by sequencing the bacterial 16S rRNA gene (V1-V2 region). Taxa with mean abundance $>1 \%$ were compared using Wilcoxon rank sum test, correcting for multiple comparisons. Disease severity was assessed with the Birmingham Vasculitis Activity Score for Wegener's Granulomatosis (BVAS/WG). Effects of medications were studied using mixed effects models.

Results: 63 fecal samples were studied: 29 active AAV (15 new diagnosis/14 chronic), 20 in remission, and 14 healthy controls. Compared to controls, patients with active AAV had a different microbial composition $(p=0.01)$. There was no statistical difference between the gut microbial composition of controls and patients in remission $(p=0.16)$. The relative abundance of the taxa Dialister and Prevotella were different between active and remission AAV. The relative abundance of the genera Faecalibacterium and Sutterella were different between active and remission newly-diagnosed AAV (Figure 1A). The relative abundance of Dialister was significant in patients with high BVAS/WG compared to patients with low BVAS/WG $(p<0.01)$ (Figure 1B). High BVAS/WG was associated with greater dysbiosis (Figure 2); similar results were found in a multivariate linear model $(p=0.02)$. The gut microbiome in patients with GPA on immunosuppressive agents was similar to controls $(p=0.54)$, whereas the gut microbiome of patients with GPA not on these therapies was significantly different from controls; similar results were found with glucocorticoids and antibiotics use.

Conclusion: Active AAV is associated with an altered gut microbial composition. Patients in clinical remission have microbial composition similar to healthy controls. Immunosuppressive agents, glucocorticoids, and antibiotics may re-establish a healthy gut microbiome. Severe disease activity is associated with worsening gut dysbiosis suggesting a potential role of gut bacteria in the pathogenesis of AAV.
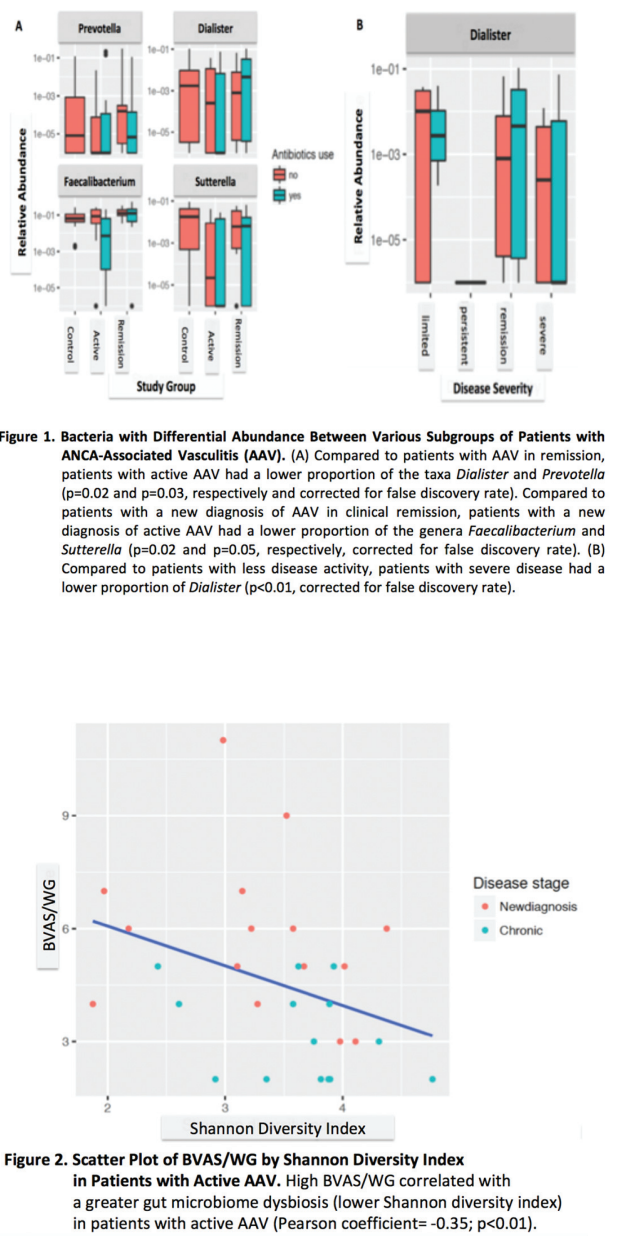

\section{REFERENCES:}

[1] Jennette J.C., et al., 2012 revised International Chapel Hill Consensus Conference Nomenclature of Vasculitides. Arthritis Rheum, 2013. 65(1): p. $1-11$.

[2] Merkel P.A., et al., Identification of functional and expression polymorphisms associated with risk for anti-neutrophil cytoplasmic autoantibody associated vasculitis. Arthritis Rheum, 2017. 69(5): p. 1054-1066.

[3] Scher J.U., et al., Microbiome in Inflammatory arthritis and human rheumatic disease. Arthritis Rheum, 2016. 68: p.35-45.

[4] Scher J.U. Intestinal dysbiosis and potential consequences of microbiomealtering antibiotic use in the pathogenesis of human rheumatic disease. The Journal of Rheum, 2015. 42: p.355-357.

[5] Scher J.U., et al., Decreased bacterial diversity characterizes an altered gut microbiota in psoriatic arthritis and resembles dysbiosis of inflammatory bowel disease. Arthritis and Rheum, 2015. 67(1): p.128-139

Disclosure of Interests: None declared

DOI: 10.1136/annrheumdis-2019-eular.8322

\section{\begin{tabular}{|l|l}
\hline FRI0684 NOVEL MAPPING FUNCTION ILLUSTRATES \\
\hline
\end{tabular} NONLINEARITY BETWEEN TRIAL ACR RESPONSE, DAS28 CHANGE AND EULAR RESPONSE CRITERIA}

Nuria Navarro Coy ${ }^{1}$, Kimme Hyrich ${ }^{2}$, Sue Pavitt ${ }^{3}$, Robert West ${ }^{4}$, Maya Buch ${ }^{1,5}$ ${ }^{1}$ Leeds Institute of Rheumatic and Musculoskeletal Medicine (LIRMM), Leeds, United Kingdom; ${ }^{2}$ Institute of Inflammation and Repair, Manchester, United Kingdom; ${ }^{3}$ School of Dentistry, Leeds, United Kingdom; ${ }^{4}$ Leeds Institute of Health Sciences, Leeds, United Kingdom; ${ }^{5}$ NIHR Leeds Biomedical Research Centre, Leeds Teaching Hospitals Trust, Leeds, United Kingdom

Background: The American College of Rheumatology (ACR) definition of improvement is a standardised, widely used outcome measure for clinical trials in rheumatoid arthritis. In routine clinical practice (and registries), 
improvement is assessed by the DAS28 (Disease Activity Score 28), particularly in the UK, where it is the basis of current NICE guidance'.

Objectives: To develop a mapping function that charts ACR20/50/70 and ACRn to $\triangle \mathrm{DAS} 28$ and EULAR response, and determine the relationship between the response measures.

Methods: Individual patient-level data from the 428 participants randomised to the ATTRACT ${ }^{2}$ trial were used. The proportion of participants with ACRn (ACR10-ACR90) response and the change in DAS28 $(\triangle \mathrm{DAS} 28)$ at 30 weeks from baseline were calculated and analysed analysed in participants with complete data. The cut points in $\triangle \mathrm{DAS} 28$ that provided the equivalent proportion of participants achieving ACR10 to ACR90 at 30 weeks overall and per individual arm were searched for through tabulation. Regression models were conducted and misclassification rates calculated to test the accuracy of the mapping function. The minimum and maximum ACRn achieved per EULAR response category were also determined to establish the correlation of these two response criteria. R version 3.4 .3 (2017-11-30) was used for all statistical analyses. Results: $53 \% / 27 \% / 12 \%$ of all trial participants achieved ACR-ESR 20/50/ 70 responses, respectively. The mapping function shows that at 30 weeks a $20 \%$ improvement in ACR is equivalent to a $27.3 \%$ and $27.8 \%$ improvement from baseline in DAS28 calculated using ESR and CRP, respectively. $50 \%$ improvement in ACR is equivalent to a $45 \%$ and $46.2 \%$ improvement in DAS28-ESR and DAS28-CRP, respectively. $70 \%$ improvement in ACR is equivalent to a lower improvement in the DAS28, at $55.8 \%$ for ESR and $59.3 \%$ for CRP. Similar results were obtained when analysing the individual arms (Table 1 \& Figure 1). Baseline DAS28 for all analysed groups was $\geq 6.0$. Moderate or good EULAR responses correspond to $80 \%$ and $90 \%$ improvement in ACR, respectively, whereas the no response EULAR category showed a maximum improvement in ACR-ESR/CRP of $30 \%-50 \%$, respectively (Figure 2).

Conclusion: This novel mapping function enables the comparison of trials reporting ACR20/50/70 with those reporting $\triangle \mathrm{DAS} 28$. The results imply nonlinearity in the relationship between ACR20/50/70, $\triangle \mathrm{DAS} 28$ and EULAR response criteria. Limitations: The mapping is dependent on the population chosen to examine it, which in this analysis comprised trial participants with very active disease.

\section{REFERENCES: :}

[1] National Institute for Health and Care Excellence (NICE), Technology Appraisal Guidance (TA375) 2016. 2Maini R, et al. Lancet 1999.

Table 1. Mapping between the ACR criteria and change in DAS28

\begin{tabular}{|c|c|c|c|}
\hline & Full cohort $(n=428)$ & Placebo arm $(n=88)$ & $\begin{array}{c}\text { IFX arm* }(n=86) \\
(3 \mathrm{mg} / \mathrm{kg} \text { every } 8 \text { weeks })\end{array}$ \\
\hline & $\Delta$ DAS28-ESR (\%) & $\Delta$ DAS28-ESR (\%) & $\Delta$ DAS28-ESR (\%) \\
\hline ACR-ESR criteria & (Accuracy (\%)) & (Accuracy (\%)) & (Accuracy (\%)) \\
\hline $20 \%$ improvement & $27.3(80)$ & $24(85)$ & $29.5(81)$ \\
\hline $50 \%$ improvement & $45(85)$ & $39(94)$ & $44(84)$ \\
\hline $70 \%$ improvement & $55.8(91)$ & $44(91)$ & $57.9(86)$ \\
\hline ACR-CRP criteria & $\begin{array}{l}\text { DDAS28-CRP (\%) } \\
\text { (Accuracy (\%)) }\end{array}$ & $\begin{array}{c}\Delta \text { DAS28-CRP (\%) } \\
\text { (Accuracy (\%)) }\end{array}$ & $\begin{array}{c}\triangle \text { DAS28-CRP (\%) } \\
\text { (Accuracy (\%)) }\end{array}$ \\
\hline $20 \%$ improvement & $27.8(83)$ & $27(80)$ & $30(82)$ \\
\hline $50 \%$ improvement & $46.2(84)$ & $44(92)$ & $47.5(84)$ \\
\hline $70 \%$ improvement & $59.3(93)$ & $50.8(92)$ & $50.8(84)$ \\
\hline
\end{tabular}

\section{ESR (erythrocyte sedimentation rate); CRP (C-reactive protein concentration).}

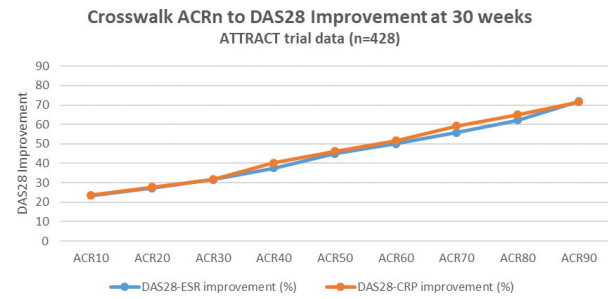

Figure 1. Mapping between ACRn (ACR10-ACR90) and DAS28 improvement at 30 weeks.

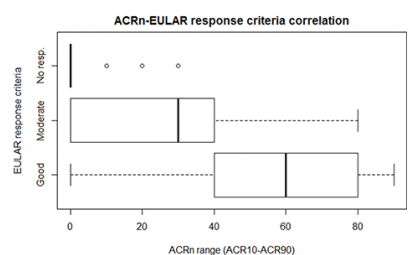

Figure 2. Correlation of the EULAR and ACR response criteria at 30 weeks.

Disclosure of Interests: Nuria Navarro Coy: None declared, Kimme Hyrich Grant/research support from: Grants to institution: BMS, Pfizer, UCB, Sue Pavitt: None declared, Robert West: None declared, Maya Buch Grant/ research support from: Pfizer LTD, UCB, Consultant for: AbbVie, Eli Lilly, EMD Serono, Pfizer Ltd., Sanofi

DOI: 10.1136/annrheumdis-2019-eular.4642

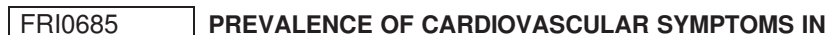 PATIENTS WITH IDIOPATHIC INFLAMMATORY MYOPATHIES - A QUESTIONNAIRE BASED STUDY}

Aleksandra Opinc, Olga Brzezińska, Joanna Makowska. Medical University of Lodz, Department of Rheumatology, Lodz, Poland

Background: Cardiovascular involvement in patients with idiopathic inflammatory myopathies (IIM) significantly worsen the prognosis and remain one of the major cause of death ${ }^{1}$. Although it is usually subclinical, symptomatic manifestations are not infrequent and exert considerable impact on the course of $1 \mathrm{IM}^{2}$.

Objectives: To evaluate the frequency of subjective symptoms indicating cardiovascular involvement in patients with different subtypes of IIM and assess the impact of such ailments on daily living and degree of disability.

Methods: An online questionnaire was created and distributed by means of online support groups to English-speaking patients with IIM. Responders were asked to answer 40 questions concerning subtype and duration of disease, cardiac and musculoskeletal symptoms as well as to fill the Short 2-page Health Assessment Questionnaire (HAQ). Responses of patients with dermatomyositis (DM), polymyositis (PM), inclusion body myositis (IBM) and overlapping syndrome (OS) were analysed statistically. Results: Questionnaire was completed by 377 responders, among which $38,78 \%$ had IBM, 34,07\% DM, 16,62\% PM and 10,53\% OS. Dyspnoea at rest affected at least a few times a month $48,33 \%$ patients with PM, $43,09 \%$ with DM, 28,95\% with OS but only $20,71 \%$ with IBM (PM vs OS $p=0,057$, IBM vs $D M p<0,001$, IBM vs $P M p<0,001)$. Dyspnoea during daily activities was declared by $55,26 \%-60,98 \%$ of patients with OS, PM and DM, but only $37,14 \%$ with IBM (IBM vs OS $\mathrm{p}=0,04$, IBM vs DM $p<0,001$, IBM vs $P M p=0,003)$. Dyspnoea at exercise was more prevalent in $\mathrm{PM}$ and DM groups (respectively $80 \%$ and $86,18 \%$ vs $71,05 \%$ and $52,14 \%$ in OS and IBM; DM vs OS $\mathrm{p}=0,03$, IBM vs OS $\mathrm{p}=0,04$, IBM vs DM $p<0,001$, IBM vs PM $p<0,001)$. Chest pain was noted mostly by participants with DM, OS and PM as in $38,33 \%-45,53 \%$ of them it occurred at least a few times a month, while it affected only 1/5 of responders with IBM (IBM vs OS $p=0,002$, IBM vs $D M p<0,001$ ). Only $37,14 \%$ of patients with IBM experienced palpitations while it was declared by $51,67 \%-63,16 \%$ of participants with PM, DM, OS (IBM vs OS $p=0,004$, IBM vs DM $\mathrm{p}<0,001$, IBM vs $P M \quad \mathrm{p}=0,056)$. In contrary to bradycardia, a feeling of too fast heart rate was common, mostly in patients with DM. Ankle oedema and dry cough occurred in respectively 43,90-60\% and $43,33-52,03 \%$ of participants. Degree of disability, assessed with HAQ was significantly higher in IBM patients. Regardless of myositis subtype dyspnoea during daily activities hindered reaching, dressing and gripping (respectively $p=0,015, p=0,03, p<0,01$ if comparing patients with and without dyspnoea). Arising, walking and maintaining hygiene was less troublesome for participants with exertional dyspnoea, chest pain, palpitations or tachycardia as compared to patients without those symptoms.

Conclusion: Symptomatic cardiovascular involvement is frequent in patients with IIM. Patients with IBM are less affected by cardiac symptoms yet present the highest degree of disability. Most of cardiac symptoms do not aggravate disability.

\section{REFERENCES:}

[1] Diederichsen LP, Cardiovascular involvement in myositis, Curr Opin Rheumatol. 2017 Nov;29(6):598-603 